\title{
EL TRABAJO COOPERATIVO EN BASE AL MODELO DEL CEREBRO TOTAL: UNA EXPERIENCIA DOCENTE
}

\author{
Marta Estrada ${ }^{1}$ \\ Universitat Jaume I \\ E-mail: estrada@emp.uji.es \\ Diego Monferrer ${ }^{2}$ \\ Universitat Jaume I \\ E-mail: $\underline{\text { dmonferr@emp.uji.es }}$ \\ Mercedes Segarra ${ }^{3}$ \\ Universitat Jaume I \\ E-mail: msegarra@emp.uji.es \\ Miguel Ángel Moliner ${ }^{4}$ \\ Universitat Jaume I \\ E-mail: amoliner@emp.uij.es
}

Material original autorizado para su primera publicación en la revista académica REDMARKA. Revista Digital de Marketing Aplicado.

https://doi.org/redma.2014.01.012.4818

Recibido: 22 Abril 2014

Aceptado 15 Junio 2014

\section{Resumen}

Las exigencias planteadas en el Espacio Europeo nos llevan a plantearnos fórmulas que fomenten el aprendizaje cooperativo en el aula. En este trabajo

${ }^{1}$ Profesora Ayudante Doctor, Departamento de Administración de Empresas y Marketing Universitat Jaume I

${ }^{2}$ Profesor Contratado Doctor, Departamento de Administración de Empresas y Marketing Universitat Jaume I

3 Profesora Titular de Universidad, Departamento de Administración de Empresas y Marketing Universitat Jaume I

${ }^{4}$ Catedrático de Universidad, Departamento de Administración de Empresas y Marketing Universitat Jaume I 
describimos una experiencia docente en la que, basándonos en el Modelo del Cerebro Total de Herrmann (1989), se forman los equipos de trabajo (con perfiles homogéneos y heterogéneos) para el desarrollo práctico de la asignatura Técnicas de Ventas del Grado de ADE. Los objetivos a alcanzar son tres: 1) fomentar la cooperación; 2) analizar el funcionamiento, rendimiento y satisfacción de los equipos de trabajo; 3) obtener información sobre cómo diseñar equipos eficientes y optimizar sus recursos. El objetivo, en última instancia, es ajustar nuestros métodos de enseñanza a los estilos de aprendizaje de los alumnos.

Palabras clave: Estilos de aprendizaje, Modelo del Cerebro Total, Universidad

\begin{abstract}
The demands in the European Space lead us to consider ways to encourage cooperative learning in the classroom. In this paper we describe an educational experience in which, based on the Herrmann Whole Brain Model (1989), work teams (with homogeneous and heterogeneous profiles) are selected for the practical development of the subject Sales Techniques from ADE Degree. The aims to achieve are three: 1 ) to promote cooperation; 2) to analyze the operation, performance and satisfaction of work equipment; 3) to obtain information about how to design efficient teams and optimize their resources. The goal, ultimately, is to adjust our teaching methods to the learning styles of our students.
\end{abstract}

Keywords: Learning styles, Whole Brain Model, University 


\section{INTRODUCCIÓN}

La implementación de las exigencias derivadas del Espacio Europeo (EEES) de educación Superior han comportado una profunda reflexión sobre la configuración de las titulaciones universitarias y la forma de transmitir el conocimiento utilizada por el profesorado. En este contexto, El EEES defiende focalizar la atención en la formación de los estudiantes y más concretamente, en su desarrollo competencial (Cano, 2008). El nuevo enfoque docente se basa en la evaluación de competencias específicas (propias de cada asignatura) y competencias transversales (cuyo principal objetivo es la formación integral de la persona y su crecimiento personal) y cuya asunción es requisito fundamental para los alumnos de cualquier titulación (Baena y Padilla, 2012; Espinosa et al., 2006). Esta circunstancia implica una reforma substancial en las metodologías utilizadas y una apuesta clara por un aprendizaje en el que el alumno sea parte activa y protagonista en su proceso formativo, asumiendo responsabilidades que le permitan estar más preparado para enfrentarse a los avatares del mercado profesional (Baena, 2010; De Juan et al., 2008; González y García, 2007).

En este sentido, EEES pretende favorecer aquellas metodologías docentes que fomenten la planificación, desarrollo y evaluación de las competencias transversales en las que desarrollo del trabajo en equipo supone un elemento clave para modificar las estructuras de metas de grupo de clase, tradicionalmente competitiva e individualista, introduciendo una serie de elementos que favorecen la cooperación como medio principal de enseñanza. El aprendizaje cooperativo supone una interesante alternativa para organizar los procesos de enseñanza y de adquisición de las competencias. Se trata de una forma de trabajo en grupo basado en la construcción colectiva del conocimiento y el desarrollo de habilidades mixtas (aprendizaje y desarrollo personal, social y laboral), donde cada miembro del grupo es responsable de su aprendizaje y del de los restantes miembros del grupo (García y Troyano, 2010). En este sistema de aprendizaje la finalidad del producto académico no es exclusiva e individualista, sino que desplaza aquella en busca de la mejora de las propias relaciones sociales, donde para alcanzar tanto los objetivos 
académicos como los relacionales se enfatiza en la interacción grupal. Los alumnos inmersos en una metodología cooperativa generan una interdependencia positiva, interacción cara a cara, responsabilidad individual, mejoran sus habilidades sociales y desarrollan un procesamiento grupal autónomo (Johnson y Johnson, 1989). Este tipo de aprendizaje es más profundo y duradero (Gil, et al., 2006) ya que el alumno es parte activa y protagonista en su proceso formativo, asumiendo responsabilidades que le permitan estar más preparado para enfrentarse a los avatares del mercado profesional (Baena, 2010; De Juan et al., 2008; González y García, 2007). Estos razonamientos han motivado que diversos especialistas de educación postulen la necesidad de introducir el trabajo en equipos cooperativos dentro de la dinámica habitual de trabajo en el aula (Espinosa et al., 2006). Sin embargo, existen investigaciones que demuestran que la práctica de agrupar a los estudiantes no necesariamente genera un espacio de construcción colectiva, ya que esto depende de las estrategias y contenidos que se trabajan dentro del aula y de los perfiles de los alumnos integrados en el equipo (Pulido de Bazán y Contrera, 2005). Para propiciar el trabajo cooperativo no basta con poner a trabajar a los alumnos en torno a una mesa, es necesario conocer su forma de aprender, el sistema de selección, de procesamiento y la forma de empleo de la información de los mismos. El logro de un buen proceso de enseñanza-aprendizaje redunda en la óptima conjunción entre la metodología a utilizar y el proceso de aprendizaje de los alumnos (Pulido de Bazán y Contrera, 2005). El descubrimiento de las capacidades individuales permitirá, siguiendo planteamientos gestalianos, que el grupo salga potenciado por la suma de las particularidades de cada uno de sus integrantes.

Las diversas teorías o modelos sobre los estilos de aprendizaje: la Teoría de Sperry (1973), la Teoría del Cerebro Triuno (MacLean, 1990), el Modelo de las Inteligencias Múltiples (Gardner, 1983), el Modelo del Cerebro Total de Herrmann (1989), entre otros, resultan de obligatoria consulta al presentar diversas perspectivas sobre el funcionamiento del cerebro en los procesos de aprendizaje. En este sentido, el profesor como facilitador de los procesos pedagógicos, ha de desplegar una serie de estrategias en las que apoyándose 
de los conocimientos aportados por los modelos o teorías de aprendizaje, contribuya a la construcción del conocimiento y al mayor desarrollo de la potencialidad del grupo. Hoy sabemos que, tal y como señala Pulido de Bazán y Contreras (2005), conocer cómo aprenden nuestros alumnos y determinar cuáles son los factores que influyen sobre los procesos de aprendizaje son aspectos muy importantes en el momento de planificar el dictado de la asignatura.

En este trabajo proponemos una experiencia basada en el Modelo del Cerebro Total. Los planteamientos de esta teoría suponen un recurso interesante al permitir al profesor averiguar el perfil individual de cada estudiante, saber que capacidades de su cerebro tiene más potenciadas $y$, de esta forma, obtener información que fomente el aprendizaje cooperativo defendido en el EEES. En base a esta línea, presentamos una actividad docente realizada en la asignatura obligatoria de $4^{\circ}$ curso Técnicas de Ventas del Grado de ADE consistente en la creación de equipos de trabajo de acuerdo a los perfiles de dominancia de los alumnos matriculados. Esta experiencia tiene un triple objetivo: 1) desarrollar la competencia transversal del aprendizaje cooperativo; 2) analizar el funcionamiento, rendimiento y satisfacción de los equipos de trabajo dependiendo de si el grupo está formado por alumnos con el mismos perfil de aprendizaje (equipos homogéneos) o distinto perfil (equipos heterogéneos); 3) tomar decisiones en base a los resultados obtenidos que nos permitan diseñar los equipos en posteriores cursos así como adoptar la metodología de enseñanza más eficaz según las características del grupo.

En la redacción de este trabajo en primer lugar, describimos las teorías o modelos sobre los estilos de aprendizaje y más concretamente el Modelo del Cerebro Total, en segundo lugar relatamos la experiencia docente, analizamos los datos y resultados y finalmente aportamos las conclusiones.

\section{LAS TEORÍAS SOBRE LOS HEMISFERIOS CEREBRALES: EL MODELO DE HERRMANN}

\subsection{Teorías sobre los hemisferios cerebrales}


Los modelos sobre los estilos de aprendizaje relacionados con el funcionamiento del cerebro provienen, en sus orígenes, de los estudios sobre sus particularidades y relación con la conducta humana efectuados desde la neurociencia. En este sentido, los resultados más relevantes de la investigación consisten en el descubrimiento de dos hemisferios cerebrales que difieren significativamente en su funcionamiento. La naturaleza de esta diferencia ha sido intensivamente estudiada desde la década de los años 50 por biólogos, neurólogos y psicólogos (Gómez, 2003). Uno de los trabajos pioneros en esta área fue el realizado por Sperry (1973) y que dio origen a la Teoría del Cerebro Derecho vs. El Cerebro Izquierdo, siendo el punto de partida para otras interpretaciones sobre el funcionamiento del cerebro y el aprendizaje como la Teoría del Cerebro Triuno y el Modelo del Cerebro Total.

La Teoría de Sperry (1973) establece que los dos hemisferios controlan diferentes modos de pensamiento y que depende de cada individuo el priorizar uno sobre otro. Los resultados de estas investigaciones permitieron conocer aspectos relacionados con el control de la conducta, por ejemplo, que el lado izquierdo está controlado principalmente por el hemisferio derecho y que el lado derecho por el hemisferio izquierdo. Otras investigaciones (Gazzaniga et al., 1962) estuvieron orientadas a determinar el intercambio de información entre los hemisferios. En esta línea, el aporte más significativo es haber descubierto que los dos hemisferios son responsables de diferentes maneras de procesar la información y, por ende, de establecer diferentes estilos de pensamiento. Así, el cerebro izquierdo está especializado en el procesamiento secuencial, paso a paso. Es lógico, racional, lingüístico, objetivo y coherente. En cambio, el cerebro derecho está especializado en el proceso simultáneo o en paralelo, integra partes y las organiza en un todo. Es memorístico, espacial, sensorial, intuitivo, holístico, sintético y subjetivo (Velásquez et al., 2007).

Por otro lado, la Teoría del Cerebro Triuno (MacLean, 1990) presenta otra visión del funcionamiento del cerebro y sus implicaciones para la educación, sirviendo de complemento de la teoría anterior. MacLean considera que existen tres cerebros integrados en uno: el reptiliano, el sistema límbico, y la neocorteza. El cerebro reptiliano o básico es el responsable de la supervivencia 
del individuo, de la conducta automática o programada. El sistema límbico está formado por el cerebro afectivo, en el que se dan los estados de calidez, amor, odio y el resto de emociones. Por último, la neocorteza está formada por los dos hemisferios en donde se llevan a cabo los procesos intelectuales superiores. El hemisferio izquierdo se ocupa del razonamiento lógico mientras que el hemisferio derecho se ocupa del razonamiento abstracto.

Otro investigador, Gardner (1983), propone el Modelo de las Inteligencias Múltiples y concibe la inteligencia no como un todo unitario sino como un conjunto de inteligencias múltiples, distintas y semi-independientes. Gardner define la inteligencia como una habilidad que se puede cultivar a través de la educación y no como algo innato. Todos los individuos son capaces de conocer el mundo desde ocho inteligencias diferentes: la inteligencia lingüística, la lógico-matemática, la corporal-Kinestésica, la musical, la espacial, la naturalista, la interpersonal y la intrapersonal. La diferencia entre unos individuos y otros estriba en la intensidad de estas inteligencias y en la forma en las que se recurre a las mismas para solucionar los problemas. Los individuos aprenden, representan y utilizan el saber de muchos y diferentes modos.

Además de estos modelos, tal y como señalan otros autores como Pérez (2001) y Gómez et. al. (2010), existen otros que estudian el estilo de aprendizaje de acuerdo con criterios como: el sistema de selección de la información, tal y como mantiene la Programación Neurolingüística (estilos visual, auditivo y cinestésico), el de procesamiento de la información (estilos lógico y holístico), la forma de empleo de la información (activo, reflexivo, teórico y pragmático) (Kolb, 1984; Kolb y Kolb, 2005) o criterios mixtos, cómo el del Modelo de las Cuatro Categorías Bipolares (activo/reflexivo, sensorial/intuitivo, visual/verbal y secuencial/global) (Felder y Silverman, 1988). La revisión de los mismos muestra la importancia, tal y como se señala desde la neurociencia, de utilizar metodologías de carácter mixto que combinen técnicas secuenciales con otras que permitan el desarrollo de pensamientos visuales y espaciales, la fantasía, el lenguaje evocador y la experiencia directa. Sobre esta idea de potenciar la utilización de distintas partes de nuestro 
cerebro como método de aprendizaje eficaz se basa el Modelo del Cerebro Total de Herrmann (1989) y en el que nos centramos en este trabajo.

\subsection{El Modelo del Cerebro Total de Herrmann}

En base a los estudios sobre la Dominancia Cerebral (Sperry, 1973) y el Cerebro Triuno (MacLean, 1978), Herrmann (1989) elaboró el Modelo del Cerebro Total. En su modelo, Herrmann integra la neocorteza (hemisferio izquierdo y derecho) con el sistema límbico dividiéndolos en cuatro cuadrantes interrelacionados que constituyen modalidades autónomas de procesamiento diferencial de la información, las cuales pueden ser convenientemente desplegadas de manera individual o combinada, tanto secuencial como simultáneamente, en los diferentes procesos del funcionamiento cerebral (véase Figura 1). Cada cuadrante se especializa en la realización de unas determinadas funciones. Así, el lóbulo superior izquierdo (cuadrante A) se especializa en el pensamiento lógico-analítico, cualitativo, matemático y basado en hechos. Mientras que el lóbulo inferior izquierdo (cuadrante B), se dedica al pensamiento secuencial, organizado, planificado y detallado. Por otro lado, el lóbulo inferior derecho (cuadrante C) supone el pensamiento emocional, comunicador, sensorial, espiritual y humanístico. Por último, el lóbulo superior derecho (cuadrante D) se basa en el pensamiento conceptual, holísticointuitivo, estratégico, integrador, creativo, espacial y visual.

Con la aplicación del Instrumento de Dominancia Cerebral de Herrmann (HBDI) se detecta el perfil de combinación de cuadrantes, el cual indica qué cuadrantes son dominantes, formando así cuatro modalidades de pensamiento: 1) realista, propio del hemisferio izquierdo (cuadrantes $A$ y $B$ ); 2) idealista, propio del hemisferio derecho (cuadrantes C y D); 3) pragmático (cuadrantes $A$ y D); e instintivo (cuadrantes B y C). El individuo manifiesta una dominancia cerebral, es decir, una tendencia a utilizar más las funciones de un hemisferio que las de otro para interactuar con su medio (Salas et al., 2004), en uno/s cuadrante/s u otro/s. La mayoría de los sujetos procesa la información desde varios cuadrantes. El HBDI permite determinar el perfil distintivo de los individuos estableciendo la dominancia según las puntuaciones alcanzadas en 
el test. En este sentido, una puntuación superior a los 67 puntos implica una clara dominancia en un determinado perfil, dominancia primaria; entre 34 y 66 puntos, supone una dominancia secundaria o indecisión; y menos de 34 puntos, dominancia terciaria o rechazo. Los perfiles de los cuadrantes se representan por los números 1,2 y 3 , de acuerdo con la puntuación obtenida por cada uno, en la secuencia A, B, C, D. De esta manera, el perfil 1-2-3-2, por ejemplo, significa que hay dominancia primaria en el cuadrante $A$, dominancia secundaria en el B, terciaria en el $C$ y secundaria en el D. El perfil 1-1-1-1 significa que se tiene una dominancia primaria en todos los cuadrantes (cuádruple dominancia), en el extremo opuesto se tiene el perfil 3-3-3-3 (cuádruple rechazo) y el perfil 2-2-2-2 significa que no hay dominancia ni rechazo por ninguno de los cuadrantes. Existen entonces, bajo todas las combinaciones posibles, los siguientes grados de dominancias: simple, doble, triple y cuádruple. Partiendo de una muestra de más de 500.000 sujetos Herrmann observa que sólo el $6 \%$ de los individuos tienen una dominancia simple. El 60\% tiene una dominancia doble y procesa la información desde dos cuadrantes siguiendo alguna de las siguientes combinaciones $A B ; C D ; A D ; A C$ y BC. El 30\% tiene una dominancia triple y genera sus pensamientos desde las combinaciones $\mathrm{ABC}, \mathrm{BCD}, \mathrm{CDA}, \mathrm{DAB}$. Por último, una minoría compuesta por el 3\% de los individuos tiene dominancia cuádruple.

Figura 1: El Modelo del Cerebro Total

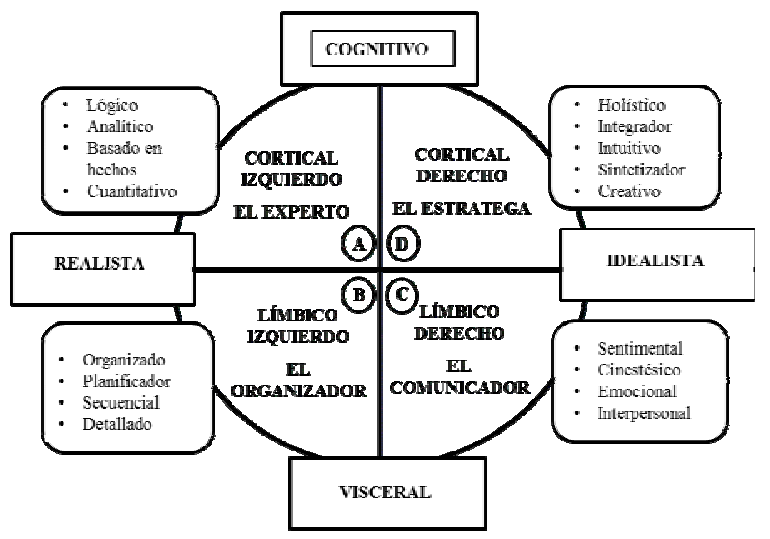

Fuente: Basado en Cazau (2004). 
El individuo con dominancia simple en el cuadrante A (cortical izquierdo) es frío, de pocos gestos, intelectualmente brillante, capaz de evaluar y criticar, competitivo e individualista. Los procesos cognitivos que desarrolla son: el análisis, el razonamiento, la lógica, el rigor, la claridad, el gusto hacia los modelos y las teorías, colecciona hechos y prefiere la palabra precisa. Las estrategias de procesamiento de información que conforman este cuadrante constituyen la referencia básica de la enseñanza, la formación científica y la capacitación profesional en aquellas especialidades asociadas con el rigor del pensamiento analítico y cuantitativo. Las competencias que posee son la abstracción, las matemáticas, las finanzas y la resolución de problemas (Velásquez et al., 2007). Este cuadrante se asocia el esfuerzo constante, la experiencia y la capacitación profesional.

El individuo con dominancia simple en el cuadrante B (límbico izquierdo) es introvertido, emotivo, maniático, tiende a monologar, es secuencial, metódico, es conservador y fiel. Los procesos cognitivos que desarrolla son la planificación, la formulación, la estructuración y la definición de procedimientos. Las estrategias que conforman el cuadrante B pueden ser definidas como operacionales y dirigidas fundamentalmente a la ejecución de acciones previamente diseñadas (no concibe la búsqueda de alternativas distintas a las pautadas), lo importante es administrar recursos y supervisar el desempeño de las metas. Si un individuo con dominancia en el cuadrante B no cuenta con elementos suficientes para la planificación, la ejecución y la evaluación de procesos se hace difícil alcanzar la eficiencia y la calidad deseada. Las competencias que posee son: la administración, la organización y la capacidad de liderazgo (Velásquez et al., 2007).

El individuo con dominancia simple en el cuadrante C (límbico derecho) es extrovertido, emocional, espontáneo, gesticulador, lúdico, hablador, espiritual y reacciona negativamente hacia las críticas. Los procesos cognitivos que desarrolla son: la integración mediante la experiencia, la tendencia hacia el placer, la fuerte implicación afectiva y la escucha activa, siente la necesidad de compartir y evalúa los comportamientos. Presenta una clara tendencia a involucrarse en relaciones interpersonales de cooperación y amistad, en 
proyectos sociales y comunitarios y en causas humanitarias. Las competencias que posee son: la relación interpersonal, la propensión al diálogo, el gusto por la docencia, la tendencia al trabajo en equipo y la comunicación (Velásquez et al., 2007).

El individuo con dominancia simple en el cuadrante D (cortical derecho) es original, con sentido del humor, gusto por el riesgo, discutidor, futurista, de discurso brillante e independencia. Los procesos cognitivos que desarrolla son: conceptualización, síntesis, imaginación, visualización, asociación, integración por imágenes. Sus principales competencias son la innovación, la creación, el espíritu empresarial y la investigación (Velásquez et al., 2007).

En este trabajo, nos basamos en el Modelo de Cerebro Total para comprender el estilo de aprendizaje individual utilizado por los alumnos y diseñar fórmulas óptimas que sirvan para la mejora del aprendizaje cooperativo de los trabajos en grupo, potenciando así el rendimiento académico. En este sentido, analizaremos qué perfiles y dominancias pueden cooperar de forma más eficaz y lograr la mayor satisfacción individual.

\section{DESCRIPCIÓN DE LA ACTIVIDAD DOCENTE}

La cooperación, comparada con esfuerzos competitivos e individualistas tiende a resultar, en altos niveles de logro, una mayor retención a largo plazo de lo aprendido, un uso más frecuente de niveles altos de razonamiento y una mayor voluntad para persistir en tareas difíciles (Gil et al., 2006). Además, implica una mayor motivación intrínseca y una mayor facilidad para transferir el aprendizaje de una situación a otra. Sin embargo, tal y como se ha descrito en apartados anteriores, para el logro del éxito del trabajo en equipo resulta fundamental la elección correcta de los perfiles del estilo de aprendizaje de sus miembros. En este sentido se plantea el trabajo que a continuación se detalla.

\subsection{Objetivos}


En el contexto descrito, el objetivo más inmediato de esta experiencia es que los alumnos trabajen de manera colaborativa a través de un proceso de conocimiento compartido en el que el alumno sea capaz de comprender, analizar y adoptar la perspectiva del resto de los miembros del grupo, llegar a acuerdos y encontrar soluciones y resultados compartidos en aras a alcanzar un objetivo común. Esta forma de trabajar genera un sentimiento de interdependencia, confianza y responsabilidad en el grupo que fomenta la interacción de sus miembros en la construcción conjunta de ideas y, por tanto, en la configuración conjunta del trabajo final encargado por el profesor. Para alcanzar este objetivo nos proponemos:

- Desarrollar en el alumnado, al margen de su perfil de dominancia y desde la cooperación, las habilidades conceptuales necesarias para superar con éxito la asignatura.

- Fomentar la habilidad procedimental del trabajar en grupo. Esto implica fomentar en el alumno las destrezas necesarias para la negociación, la correcta planificación, el diálogo, la asertividad y la empatía para aprender del perfil de los compañeros.

- Potenciar la cooperación entre compañeros con diversos perfiles de dominancia.

A este objetivo general se suman dos objetivos cuyo logro se sitúan más en el corto y medio plazo y que también son propósito de esta experiencia docente:

- Conocer el grado de satisfacción percibido por el alumnado en su relación con el equipo al que ha sido asignado y con el que ha trabajado.

- Obtener información sobre los perfiles de dominancia de la titulación y cuáles son las combinaciones más adecuadas para obtener los mejores resultados en el trabajo cooperativo generado en esta asignatura. Esto nos permitirá incrementar la efectividad en el diseño de los equipos del próximo curso, así como adoptar la metodología de enseñanza a las características del grupo. 


\subsection{Metodología}

Para llevar a cabo este proyecto se utiliza una muestra de conveniencia de 84 sujetos, 39 hombres y 45 mujeres, con edades comprendidas entre los 22 y los 30 años, estudiantes de la asignatura Técnicas de Ventas, obligatoria de $4^{\circ}$ curso del Grado en Administración de Empresas de la Universitat Jaume I, impartida durante el primer semestre del curso 2013-2014.

La metodología utilizada para la valoración del aprendizaje cooperativo se fundamenta en el método del caso y en la realización de un trabajo final en equipo consistente en la redacción de un manual de ventas de una empresa de libre elección.

Los instrumentos de valoración para el grado de consecución de los objetivos propuestos son:

1) El sistema de evaluación de la asignatura a través de la nota académica. Dicho sistema nos permite obtener información sobre el éxito de la metodología utilizada. En este sentido, la evaluación se compone de un examen teórico/práctico (50\% de la puntuación) al que hay que sumarle la nota obtenida en la parte práctica de la asignatura. Las prácticas se desarrollan en equipo y suponen la resolución de casos prácticos (20\% de la puntuación) y la redacción de un manual de ventas (30\% de la puntuación).

2) El cuestionario elaborado por Jiménez (2006) que tiene su antecedente inmediato en el Herrmann Brain Dominance Instrument (HBDI) y que permite la obtención del perfil de dominancia de cada alumno y, por lo tanto, posibilita la formación posterior de equipos de trabajo (homogéneos y heterogéneos).

3) El cuestionario elaborado por Viles et al. (2013) para medir la satisfacción del alumno con el equipo asignado.

\subsection{Cronograma de acción}

El desarrollo de esta experiencia docente se distribuye y temporaliza en las siguientes fases: 


\section{Fase 1: Formación del profesorado.}

Durante el segundo semestre del curso 2012-2013 los profesores de la asignatura, tanto de la parte teórica como de la práctica, asisten a un curso organizado por la Unidad de Soporte Educativo de la Universitat Jaume I sobre modelos de aprendizaje. Como resultado del mismo surge la creación de un Seminario Permanente de Innovación Educativa (SPIE) cuyo objetivo es aprender a formar equipos de trabajo en el aula.

Fase 2: Planificación del proyecto.

Al comienzo del semestre se establecieron una serie de reuniones entre el profesorado con el fin de plantear objetivos comunes, discutir sobre el instrumento de medida idóneo y establecer reparto de responsabilidades y el calendario de acciones.

Fase 3: Respuesta al cuestionario sobre los cuadrantes cerebrales por parte de los alumnos.

Para realizar la medición y evaluación respecto a los estilos de aprendizaje dominantes de los estudiantes, el instrumento de medida utilizado fue el elaborado por Jiménez (2006) que tiene su antecedente inmediato en el Herrmann Brain Dominance Instrument (HBDI), instrumento de papel y lápiz elaborado por Herrmann (1989), ampliamente utilizado por su autor en muestras norteamericanas, y elemento clave en la conformación de su Modelo del Cerebro Total.

Concretamente, este instrumento de 40 ítems (ver Tabla 1), supone un proceso de auto-evaluación en el que el entrevistado debe mostrar, en una escala tipo Likert de 5 puntos (donde 1 es "lo que hago peor" y 5 es "lo que hago mejor"), su opinión respecto a su grado de desempeño en cada uno de los aspectos o actividades asociados a los distintos cuadrantes cerebrales (10 ítems asociados a cada cuadrante). La valoración total en cada cuadrante se obtiene multiplicando por dos los resultados obtenidos en cada uno de ellos. 
Tabla 1: Escala de medición para el diagnóstico del Modelo del Cerebro Total CUADRANTE A (superior izquierdo cerebral)

1. Tengo habilidades específicas en el campo de las matemáticas y las ciencias.

2. Pienso que la mejor forma de resolver un problema es siendo analítico.

3. Me inclino hacia la crítica en todos los asuntos.

4. Tengo habilidades para solucionar problemas complejos de manera lógica.

5. Antes de tomar algo como verdadero, lo compruebo, e indago otras fuentes.

6. Tengo capacidad de comprender, manipular números y estadísticas de acuerdo con un fin.

7. Me gusta solucionar problemas inclinándome a conocerlos y buscar mediciones exactas.

8. Tengo la capacidad frente a los problemas de razonar en forma deductiva, a partir de alguna teoría.

9. Descompongo ante un problema las ideas y las relaciono con la totalidad.

10. Selecciono alternativas sobre la base de la racionalidad y la inteligencia, en oposición al instinto, a la emoción.

\section{CUADRANTE B (inferior izquierdo límbico)}

11. La planificación y la organización son prioritarias en mis actividades.

12. Es importante para mí tener un lugar para cada cosa y cada cosa en su lugar.

13. Acostumbro escuchar las opiniones de los demás y hacer aclaraciones.

14. Prefiero las instrucciones específicas en lugar de aquellas generales que dejan muchos detalles opcionales.

15. Pongo mucha atención en los pequeños detalles o partes de un proyecto.

16. Tengo capacidad de control y dominio de mis emociones cuando elaboro un plan o proyecto.

17. Pienso que trabajar con un método paso a paso es la mejor manera de resolver mi problema.

18. Tengo habilidades específicas en el manejo de auditorio o hablar en público.

19. Formulo métodos o medios para alcanzar un fin deseado antes de pasar a la acción.

20. Tengo la capacidad de coordinar a las personas o de ordenar los elementos para lograr relaciones coherentes y armoniosas.

CUADRANTE C (inferior derecho límbico)

21. Prefiero trabajar en equipo que hacerlo sólo.

22. Es importante para mí estar en muchas oportunidades acompañado.

23. Creo en la trascendencia humana, en algo superior o espiritual.

24. Soy emotivo frente a las situaciones difíciles.

25. A menudo actúo para solucionar problemas de tipo social.

26. En muchas ocasiones prima más en mis decisiones, lo emotivo que lo lógico y lo racional.

27. Disfruto, observo y me emociono frente a la belleza de la naturaleza.

28. Tengo habilidades para percibir, entender, manipular posiciones relativas de los objetos en el espacio.

29. Utilizo todos mis sentidos con frecuencia para resolver problemas (olfato, vista, gusto, tacto, oído).

30. Tengo la capacidad de desarrollar y mantener buena comunicación con diferentes tipos de personas.

CUADRANTE D (superior derecho cerebral)

31. Tengo un interés muy fuerte o talento con la música, la poesía, la escultura. También para pintar, dibujar, esquematizar etc.

32. Tengo la capacidad de razonar en forma avanzada y creativa, siendo capaz de adquirir, modificar y retener conocimientos.

33. Produzco nuevas ideas e innovaciones en mi trabajo.

34. Tengo la capacidad de entender y hacer uso de imágenes visuales y verbales para representar semejanzas y diferencias.

35. Tengo la capacidad de percibir y entender una problemática global sin entrar en el detalle de los elementos que la componen.

36. A menudo mis mejores ideas se producen cuando no estoy haciendo nada en particular.

REDMARKA UIMA-Universidad de A Coruña - CIECID

101

Año VII, Número 12, (2014), v I pp. 87-112

http://www.redmarka.org/

ISSN 1852-2300 
37. Prefiero ser conocido y recordado como una persona imaginativa y fantasiosa.

38. Frecuentemente me anticipo a la solución de los problemas.

39. Tengo la capacidad de utilizar o comprender objetos, símbolos y señales complejas.

40. Utilizo el juego y el sentido del humor en muchas de mis actividades.

Fuente: Jiménez (2006).

Fase 4: Construcción de equipos de trabajo.

En base a los resultados obtenidos en el cuestionario de Jiménez (2006) se construyen los equipos de trabajo. En este sentido se realizan un total de 14 equipos con 6 integrantes cada uno, 8 equipos heterogéneos con perfiles de dominancias mixtas y 5 equipos homogéneos con perfiles de dominancia pura, 5 del hemisferio izquierdo y 1 del derecho. A los alumnos no se les notifica su perfil de dominancia con el fin de no sesgar su compartimiento frente al equipo.

Fase 5: Planteamiento de los casos prácticos por parte del profesorado.

Los profesores diseñan los casos prácticos a resolver por equipo. En este sentido, se plantean 6 casos representativos de todos los estilos de aprendizaje, 3 más adaptados a las características propias del hemisferio izquierdo (su optima resolución necesita de la capacidad analítica, planificadora y secuencial) y 3 con un perfil más propio del hemisferio derecho (su optima resolución necesita de la capacidad holística, creativa, intuitiva, sintetizadora, emocional para su resolución).

Fase 6: Toma de contacto del alumno con su equipo: LA ISLA.

Para conseguir la aproximación y conocimiento de los estudiantes con el equipo se realiza la práctica de LA ISLA. Esta consiste en que, a diversos equipos, una vez dotados de material propio de manualidades y de plástica (colores, pegamento, cartulina, plastilina), se les pide la ejecución de lo que entienden por el concepto isla (ver Figura 2). Esta actividad didáctica permite ver un diferente desarrollo según el perfil dominante en el grupo. Además genera relaciones interpersonales, habilidades comunicativas, crítica constructiva, creatividad, empatía y asertividad, habilidades, todas ellas, propias del desarrollo de una metodología cooperativa. A continuación observamos algunos ejemplos de las islas realizadas. 
Figura 2: La Isla
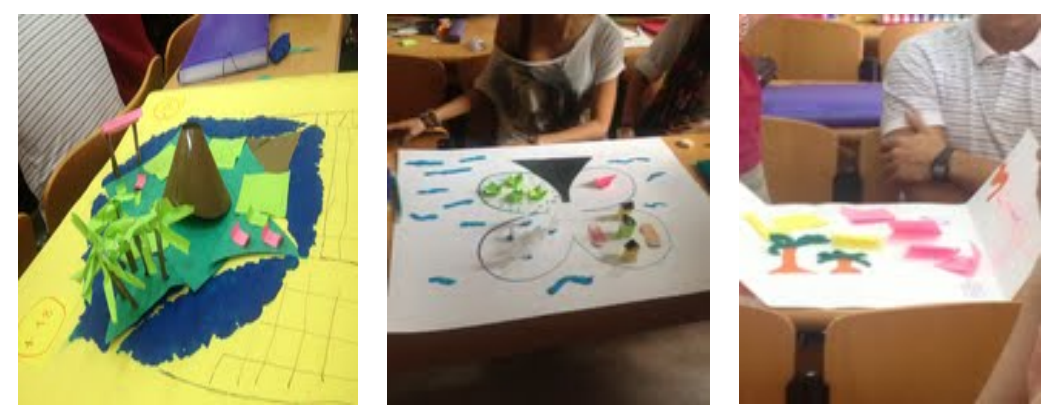

\section{Fase 7: Resolución de casos prácticos en el aula}

Los alumnos resuelven y discuten de forma cooperativa los casos en la propia aula y reciben el feedback del profesor y de la crítica constructiva resto de equipos.

\section{Fase 8: Reunión de control}

Los profesores, hacia la mitad del semestre, se reúnen para discutir la marcha de los equipos y la metodología utilizada.

\section{Fase 9: Presentación de los manuales de venta}

A través de dos sesiones prácticas, los equipos exponen públicamente los manuales de venta. En esta fase, para la evaluación de los mismos, se invita al director comercial de una importante empresa del sector azulejero para que ayude al profesor en las tareas de asesoramiento y consulta.

\section{Fase 10: Respuesta a cuestionario sobre la satisfacción grupal}

En relación a la medición de la satisfacción del alumno con respecto a su equipo de trabajo recurrimos a la escala de medida desarrollada por Viles et al. (2013). Este instrumento de medida se compone de un total de 23 ítems (ver Tabla 2), en el que el alumno debe mostrar, en una escala tipo Likert de 5 puntos, su grado de acuerdo con una serie de afirmaciones relacionadas con el funcionamiento de su grupo de trabajo a lo largo del semestre (donde 1 es "totalmente en desacuerdo" y 5 es "totalmente de acuerdo") sobre una serie de dimensiones fundamentales: la participación en la toma de decisiones (3 
ítems), la gestión de conflictos (2 ítems), la resolución de problemas (2 ítems), la cooperación (3 ítems), el liderazgo (2 ítems), la comunicación interna (3 ítems) y el feedback (8 ítems).

Tabla 2: Escala de medición para el diagnóstico de la satisfacción grupal PARTICIPACIÓN EN LA TOMA DE DECISIONES

1. Se han establecido normas internas que han facilitado el trabajo en equipo.

2. Todos los miembros han participado en las tareas del equipo.

3. Las decisiones en el equipo se han tomado teniendo en cuenta la opinión de todos los miembros.

\section{GESTIÓN DE CONFLICTOS}

4. Las discrepancias en el equipo han permitido considerar nueva ideas o nuevos puntos de vista.

5. No ha habido conflictos de tipo interpersonal y si los ha habido, se han resuelto sin que nadie se haya sentido perjudicado.

RESOLUCIÓN DE PROBLEMAS

6. Se han utilizado los datos o se ha seguido algún tipo de método para la resolución del caso planteado.

7. Se ha potenciado la creatividad para la resolución de los problemas o propuestas planteadas.

\section{COOPERACIÓN}

8. Las tareas y actividades concretas que cada miembro del equipo debería realizar han estado claras desde el inicio tanto en contenido como en plazo.

9. Tus compañeros han contribuido al equipo tal y como se estableció y ha sido necesario.

10. Ha existido colaboración entre los miembros del equipo (nos hemos ayudado, compartido información, comunicado las dificultades, etc...).

\section{LIDERAZGO}

11. El líder del equipo ha sido aceptado por todos los miembros del equipo.

12. El líder del equipo ha dirigido y coordinado las actividades del equipo.

\section{COMUNICACIÓN INTERNA}

13. El trabajo del equipo ha transcurrido en un ambiente de confianza.

14. A pesar de las diferencias entre los miembros del equipo, ha existido un ambiente de respeto entre todos.

15. En general, la comunicación ha sido buena entre los miembros del equipo.

\section{FEEDBACK}

16. Los objetivos se han transmitido bien al equipo y han sido comprendidos.

17. El profesor ha facilitado los recursos (información, materiales, tiempo, o de otro tipo) que el equipo ha necesitado.

18. Las tareas y actividades concretas a realizar por el equipo han estado claras tanto en contenido como en plazo.

19. Los criterios de evaluación se han transmitido bien al equipo y han sido comprendidos al inicio del trabajo.

20. Como equipo, hemos tenido acceso a la información que hemos necesitado.

21. En caso de necesidad, el equipo ha podido comunicarse fácilmente con los profesores de las asignaturas involucradas.

22. Como equipo, hemos recibido información acerca del resultado de nuestro trabajo (feedback del trabajo realizado, fallos cometidos, puntos a destacar).

23. El trabajo ha sido de alguna manera valorado públicamente al resto de la clase.

Fuente: Viles et al. (2013).

\section{Fase 11: Elaboración de las conclusiones}


Finalizado el curso los profesores se reúnen para analizar los resultados obtenidos y el grado de satisfacción manifestado por los alumnos con la metodología utilizada. Los análisis, resultados y conclusiones permiten observar el grado de consecución de los objetivos propuestos en esta experiencia. Además, los mismos, serán de utilidad para formar equipos de trabajo óptimos en la asignatura Técnicas de Ventas en el próximo curso.

\section{RESULTADOS}

Los datos fueron analizados mediante técnicas de análisis descriptivo y de frecuencias, análisis de la varianza (ANOVA) y análisis de varianza múltiple (MANOVA) utilizando el programa estadístico SPSS 18.0, con tal de analizar al estudiante tanto de forma individual como a nivel de equipo.

\subsection{Análisis atendiendo a criterios individuales}

En primer lugar, tal y como se recoge en la Tabla 3, se lleva a cabo un análisis descriptivo respecto a los valores promedio de cada uno de los cuadrantes así como de los perfiles de dominancia observados en los estudiantes.

Tabla 3: Análisis descriptivo de las dominancias cerebrales de la muestra

\begin{tabular}{|c|c|c|c|c|}
\hline Cuadrantes & A & B & C & D \\
\hline Media & 65,46 & 70,24 & 63,93 & 60,95 \\
\hline Desv. típica & 10,15 & 9,03 & 10,64 & 13,39 \\
\hline Dominancias & $\%$ (muestra) & $\%$ (muestra) & $\%$ (muestra) & $\%$ (muestra) \\
\hline Terciaria (0 - 33) & $0,0 \%(0)$ & $0,0 \%(0)$ & $1,2 \%(1)$ & $4,8 \%(4)$ \\
\hline Secundaria (34 - 66) & $53,6 \%(45)$ & $42,9 \%(36)$ & $61,9 \%(52)$ & $64,3 \%(54)$ \\
\hline Primaria (67 - 100) & $46,4 \%(39)$ & $57,1 \%(48)$ & $36,9 \%(31)$ & $31,0 \%(26)$ \\
\hline \multicolumn{2}{|c|}{ Dominancias primarias } & $\%$ & Muestra \\
\hline \multicolumn{2}{|r|}{ Sin dominancias primarias } & $17,9 \%$ & 15 \\
\hline \multicolumn{2}{r|}{ Dominancia hemisferio izquierdo } & $34,5 \%$ & 29 \\
\hline \\
Dominancia hemisferio derecho & $30,7 \%$ & 9 \\
\hline
\end{tabular}

Atendiendo a los valores promedio de cada uno de los cuadrantes, los alumnos encuestados presentan dominancias primarias en el cuadrante B $(70,24)$. De hecho, observamos que, al valorar la distribución en cuanto a la importancia de cada dominancia (primaria, secundaria y terciaria) para cada uno de los cuadrantes, salvo en este cuadrante $B$ en el que el mayor porcentaje es de dominancia primaria $(57,1 \%)$, en el resto de cuadrantes el mayor peso lo tienen 
las dominancias secundarias. Así, en general los alumnos analizados pertenecientes al Grado de Administración de Empresas presentan una dominancia simple en el cuadrante B (2122). Las personas con dominancia simple se comportan de forma previsible y la coherencia es un rasgo dominante. Sin embargo, pueden tener conflictos externos con personas que no tienen esta dominancia. Éste es un caso que podemos considerar atípico puesto que lo normal es encontrar dominancias dobles o triples.

Centrándonos en las posibles combinaciones de dominancias primarias existentes en la muestra vemos que, a pesar de que como suele ser habitual la mayoría de los estudiantes presentan una dominancia mixta (36,9\%), su importancia es compartida con aquellos estudiantes que presentan dominancias en el hemisferio izquierdo (34,5\%). De hecho, atendiendo a los valores de la Tabla 3 podemos ver como los cuadrantes $C$ y $D$ son los que presentan los valores medios más bajos (63,93 y 60,95 respectivamente), así como los menores porcentajes de dominancia primaria $(36,9$ y 31,0$)$.

Tomando como punto de partida estas valoraciones, relacionadas con las dominancias cerebrales de los estudiantes, se plantean diferentes cuestiones que nos permiten detectar el carácter diferenciador que los perfiles de dominancia pueden tener sobre los equipos de trabajo.

\subsection{Análisis atendiendo a criterios grupales de los equipos de trabajo}

Finalmente, centramos nuestros análisis en diversas cuestiones relacionadas con el ámbito del equipo, tratando de determinar la existencia de posibles diferencias en su rendimiento en función de sus perfiles de dominancia.

¿Existen diferencias en los resultados del equipo en base a sus perfiles de dominancia?

Para contrastar el rendimiento final del equipo en función del perfil de dominancia se revisan los 14 equipos de trabajo de los alumnos a lo largo del curso procediendo a su catalogación (tanto respecto a cuadrantes como a hemisferios cerebrales). Concretamente, realizamos diversos análisis asociados a las diferentes tipologías de trabajo realizadas por los equipos a lo largo del curso. Por un lado, el proyecto semestral consistente en el desarrollo de un manual de ventas y, por otro lado, los casos semanales resueltos en el aula. En este último aspecto, se diferencian aquellos casos de tipología más cercana al hemisferio izquierdo de aquellos más cercanos al hemisferio derecho. Como puede observarse en la Tabla 4, los resultados apuntan a que, en global, los alumnos pertenecientes a equipos con dominancia en el hemisferio izquierdo (en especial con perfiles del cuadrante A) y con dominancia mixta tienen mejores notas grupales (8,46 y 8,79 respectivamente) que los alumnos pertenecientes a equipos con dominancias en el hemisferio derecho $(7,51)$.

Adicionalmente, los resultados obtenidos demuestran que la heterogeneidad de opinión propia de los equipos mixtos dificulta la capacidad de rápida resolución asociada a la metodología del caso (presentan las valoraciones más bajas en todas las notas asociadas a casos). Sin embargo, esta diversidad de 
habilidades y destrezas les llevan a conseguir las mejores valoraciones en los proyectos semestrales, en los que el tiempo disponible para llegar a una solución se incrementa.

Más aún, si atendemos a la tipología de los casos observamos una correspondencia directa entre los perfiles de los equipos y la propia naturaleza del caso. Así en los casos asociados al hemisferio izquierdo las notas más altas son las logradas por los equipos de dominancia en este hemisferio $(8,48)$, mientras que en los casos asociados al perfil derecho, hace lo propio el equipo con dominancia en tal hemisferio $(9,53)$.

Tabla 4: Perfiles de dominancia y notas de equipo

\begin{tabular}{|c|c|c|c|c|c|c|}
\hline $\begin{array}{c}\text { Perfiles de los } \\
\text { equipos } \\
\text { (cuadrantes) }\end{array}$ & $\%$ (muestra) & Nota global & Nota plan & Nota casos & Casos izq. & Casos der. \\
\hline$A$ & $7,1 \%(1)$ & 9,00 & 9,00 & 9,13 & 9,00 & 9,40 \\
\hline $\mathrm{B}$ & $28,6 \%(4)$ & 8,29 & 8,47 & 8,61 & 8,31 & 9,01 \\
\hline $\mathrm{C}$ & $7,1 \%(1)$ & 7,51 & 7,00 & 8,77 & 8,39 & 9,53 \\
\hline $\mathrm{D}$ & $0,0 \%(0)$ & - & - & - & - & - \\
\hline Mixto & $57,2 \%(8)$ & 8,79 & 9,21 & 8,36 & 7,95 & 8,87 \\
\hline \multicolumn{2}{|l|}{$\begin{array}{r}\text { ANOVA } \\
\mathrm{F} \text { (Sig.) }\end{array}$} & $\begin{array}{l}13,690 \\
(0,000)\end{array}$ & $\begin{array}{l}16,397 \\
(0,000)\end{array}$ & $\begin{array}{c}8,220 \\
(0,000)\end{array}$ & $\begin{array}{c}9,006 \\
(0,000)\end{array}$ & $\begin{array}{c}4,514 \\
(0,006)\end{array}$ \\
\hline $\begin{array}{c}\text { Perfiles de los } \\
\text { equipos } \\
\text { (hemisferios) }\end{array}$ & $\%$ (muestra) & Nota global & Nota plan & Nota casos & Casos izq. & Casos der. \\
\hline Dom. hem. izquierdo & $35,7 \%(5)$ & 8,46 & 8,60 & 8,73 & 8,48 & 9,10 \\
\hline Dom. hem. derecho & $7,1 \%(1)$ & 7,51 & 7,00 & 8,77 & 8,39 & 9,53 \\
\hline Dom. mixta & $57,2 \%(8)$ & 8,79 & 9,21 & 8,36 & 7,95 & 8,87 \\
\hline \multicolumn{2}{|l|}{$\begin{array}{c}\text { ANOVA } \\
F(\text { Sig.) }\end{array}$} & $\begin{array}{l}15,075 \\
(0,000)\end{array}$ & $\begin{array}{l}23,213 \\
(0,000)\end{array}$ & $\begin{array}{c}7,620 \\
(0,001)\end{array}$ & $\begin{array}{c}8,463 \\
(0,000)\end{array}$ & $\begin{array}{c}5,265 \\
(0,007)\end{array}$ \\
\hline
\end{tabular}

¿Existen diferencias en el funcionamiento satisfactorio del equipo en base a sus perfiles de dominancia?

Tal y como muestran los resultados recogidos en la Tabla 5, en todas las dimensiones asociadas al funcionamiento del equipo, la diversidad de opinión y heterogeneidad de criterios y habilidades que se da entre los miembros de los equipos heterogéneos o mixtos hace que el trabajo en el mismo resulte más satisfactorio que en el caso de los equipos con perfiles de dominancia pura. De hecho, conviene resaltar el caso de los equipos con cuadrante $A$ en los que la sensación de sus miembros respecto al funcionamiento del equipo resulta insatisfactoria en prácticamente todas las dimensiones consideradas (considerando el 3 como el valor umbral entre insatisfacción y satisfacción)

Tabla 5: Perfiles de dominancia y funcionamiento satisfactorio del grupo

\begin{tabular}{|c|c|c|c|c|c|c|c|}
\hline Perfil de los grupos & $\begin{array}{c}\text { Toma } \\
\text { decision } \\
\text { es }\end{array}$ & $\begin{array}{c}\text { Gestión } \\
\text { conflicto } \\
\text { s }\end{array}$ & $\begin{array}{c}\text { Resolución } \\
\text { problemas }\end{array}$ & $\begin{array}{c}\text { Coordi- } \\
\text { nación }\end{array}$ & Liderazgo & $\begin{array}{c}\text { Comun. } \\
\text { interna }\end{array}$ & Feedback \\
\hline A & 2,89 & 2,33 & 2,73 & 2,72 & 2,33 & 3,28 & 3,52 \\
\hline
\end{tabular}

REDMARKA UIMA-Universidad de A Coruña - CIECID 


\begin{tabular}{|c|c|c|c|c|c|c|c|}
\hline B & 3,92 & 2,65 & 3,81 & 4,08 & 3,73 & 4,42 & 4,17 \\
\hline C & 3,48 & 2,50 & 4,08 & 3,78 & 3,50 & 4,22 & 3,94 \\
\hline D & - & - & - & - & - & - & - \\
\hline Mixto & 3,74 & 3,03 & 3,49 & 3,98 & 4,26 & 4,46 & 4,08 \\
\hline $\begin{array}{c}\text { ANOVA } \\
\text { F (Sig.) }\end{array}$ & $\begin{array}{c}7,210 \\
(0,000)\end{array}$ & $\begin{array}{c}9,216 \\
(0,000)\end{array}$ & $\begin{array}{c}25,570 \\
(0,000)\end{array}$ & $\begin{array}{c}10,556 \\
(0,000)\end{array}$ & $\begin{array}{c}69,612 \\
(0,000)\end{array}$ & $\begin{array}{c}23,447 \\
(0,000)\end{array}$ & $\begin{array}{c}13,687 \\
(0,000)\end{array}$ \\
\hline $\begin{array}{c}\text { Perfiles de Ios } \\
\text { grupos } \\
\text { (hemisferios) }\end{array}$ & $\begin{array}{c}\text { Toma } \\
\text { decision } \\
\text { es }\end{array}$ & $\begin{array}{c}\text { Gestión } \\
\text { conflicto } \\
\text { s }\end{array}$ & $\begin{array}{c}\text { Resolución } \\
\text { problemas }\end{array}$ & $\begin{array}{c}\text { Coordi- } \\
\text { nación }\end{array}$ & Liderazgo & $\begin{array}{c}\text { Comun. } \\
\text { interna }\end{array}$ & Feedback \\
\hline Dom. hem. izquierdo & 3,66 & 2,57 & 3,53 & 3,73 & 3,37 & 4,12 & 4,00 \\
\hline Dom. hem. derecho & 3,48 & 2,50 & 4,08 & 3,78 & 3,50 & 4,22 & 3,94 \\
\hline Dom. mixta & 3,74 & 3,03 & 3,49 & 3,98 & 4,26 & 4,46 & 4,08 \\
\hline ANOVA & 0,702 & 12,075 & 5,763 & 1,273 & 30,174 & 4,977 & 1,035 \\
F (Sig.) & $(0,499)$ & $(0,000)$ & $(0,005)$ & $(0,005)$ & $(0,000)$ & $(0,009)$ & $(0,360)$ \\
\hline
\end{tabular}

\section{CONCLUSIONES}

Las nuevas metodologías propuestas por el EEES y que propician el trabajo en equipo buscan modificar la estructura de metas del grupo en clase, tradicionalmente competitiva e individualista, e introducir actividades que favorezcan el establecimiento de la cooperación como medio principal de aprendizaje. El alumno, en este proceso construye su propio conocimiento a través la interacción con compañeros de diverso perfil. Para lograr el éxito del trabajo en equipo y el establecimiento de las bases cooperativas es muy importante determinar el perfil de aprendizaje y, de esta forma, diseñar los equipos más eficaces.

En este sentido, según los resultados de nuestro estudio, el estilo de aprendizaje de los estudiantes del Grado Administración de Empresas presenta una dominancia simple en el cuadrante B y, si nos fijamos en las dominancias dobles, se observa que tanto los perfiles de dominancia mixtos $(36,9 \%)$ como los de dominancia del hemisferio izquierdo (34,5\%) tienen la misma importancia. Esto va en línea con trabajos anteriores que mantienen la existencia de una relación entre el perfil de dominancia y la preferencia en la elección de titulaciones universitarias y futura ocupación profesional. La dominancia en el cuadrante A es propia de los alumnos de titulaciones de las ciencias en general (naturales, sociales y jurídicas). El cuadrante B es dominante en el perfil de aquellos alumnos de titulaciones relacionadas con las ciencias naturales, de la administración de empresas y la contabilidad. El perfil de dominancia en el cuadrante $\mathrm{C}$ es propio de las titulaciones relacionadas con 
la enseñanza, la sociología, el periodismo, la enfermería y el trabajo social. Por último, la dominancia en el cuadrante $D$ se manifiesta en las titulaciones de publicidad, bellas artes, música o arquitectura (Herrmann, 1989). Sin embargo, el perfil de aprendizaje más completo es el que supone una dominancia en todos los cuadrantes y, por lo tanto, la máxima representación del funcionamiento del cerebro total. Encontrar alumnos con un perfil de dominancia cuádruple, tal y como sostiene Herrmann, resulta difícil pero subsanable, formando equipos completos en los que aparezcan representados los diferentes perfiles de aprendizaje.

En este sentido, con respecto al trabajo en equipo y su composición, nuestros resultados muestran que los alumnos pertenecientes a equipos homogéneos (dominancias primarias puras) alcanzan mejores resultados que los alumnos de equipos heterogéneos, siempre y cuando la práctica se ajuste a su perfil de aprendizaje. Sin embargo, en general, los equipos heterogéneos, con dominancias mixtas, presentan mayores dificultades en la solución de los casos prácticos. Esto es consecuencia directa de que los casos prácticos se solventan de forma rápida en el aula y, en este sentido, las diferencias de perfiles entre los alumnos de estos equipos ralentiza su eficacia al necesitar de mayor tiempo para organizarse, planificarse y coordinarse correctamente. Sin embargo, cuando disponen de mayor tiempo, como sucede en el caso del manual de prácticas (trabajo semestral), consiguen vencer estas dificultades y los resultados académicos son superiores. En esta línea podríamos decir que la composición idónea para trabajar en grupo en la asignatura Técnicas de Ventas y obtener el mejor rendimiento académico es aquella formada por miembros con distintos perfiles, preferentemente alumnos con el perfil de dominancia cerebral propio de la titulación (en nuestro caso el B) y perfiles de dominancia mixto, puesto que estos tienen puntos en común con los perfiles de dominancia puros (lateralizados) pero a la vez son capaces de pensar de otra forma y enfocar una situación de aprendizaje de forma distinta.

Los equipos de trabajo heterogéneos en su forma de aprender tienen como principal reto la capacidad para aprender a gestionar sus diferencias y a ser tolerantes con las distintas formas de pensar (Herrmann, 1989; Pujolàs, 2008) 
y, si lo logran, obtendrán resultados enriquecedores. Además, la formación de equipos heterogéneos supone la preparación del alumno hacia las demandas de un mercado laboral que, no sólo necesita titulados con una gran capacitación técnica, sino que puedan adaptarse a los cambios, que sean permeables y flexibles. La sociedad actual necesita profesionales interdisciplinares capaces de enfrentarse al cambio constante, de trabajar con equipos heterogéneos, adaptarse al know-how de cualquier empresa y sector empresarial. En este sentido, los profesores universitarios tenemos la responsabilidad de formar a los estudiantes no sólo en las competencias que requieren específicamente cada titulación, sino en aquellas otras que potencien su polivalencia. Los nuevos titulados deben ser formados para utilizar los dos hemisferios cerebrales, siempre que las necesidades lo requieran.

En este sentido, proponemos desarrollar metodologías de aprendizaje a partir del Modelo del Cerebro Total. Para ello, en un primer paso el profesor debe conocer el estilo de aprendizaje de los estudiantes para formar equipos heterogéneos. En segundo lugar, desarrollar actividades que incluyan distintas formas de aprender y que no estén condicionadas por el curriculum formativo de la especialidad. Es decir, presentar actividades que obliguen a los alumnos a potenciar los dos hemisferios. El objetivo es formar titulados universitarios completos, capaces de enfrentarse a los retos del mercado laboral de una forma abierta, capaces de cooperar y enriquecerse de otras perspectivas. En definitiva hay que formar grupos e individuos que supongan en sí mismos un gran cerebro total.

La principal limitación de la experiencia descrita se refiere a que su campo de aplicación se constriñe a una sola asignatura y durante un semestre concreto. No obstante, actualmente estamos trabajando en la ampliación de esta experiencia docente a otras titulaciones contando para ello con profesores de otras áreas (comunicación, periodismo e ingenierías). Esta colaboración y cooperación interdisciplinar en el seno de la universidad nos permitirá comparar resultados y llegar a conclusiones más amplias y profundas.

\section{REFERENCIAS BIBLIOGRÁFICAS}

REDMARKA UIMA-Universidad de A Coruña - CIECID Año VII, Número 12, (2014), v I pp. 87-112 
BAENA, V. (2010). "Innovación docente e identificación de inhibidores del aprendizaje en el área de empresa. Una propuesta metodológica", Espiral-Cuadernos del Profesorado, Vol. 3(6), pgs. 3-14.

BAENA, V. Y PADILLA, V. (2012). "Refuerzo y desarrollo de competencias mediante la elaboración de una campaña real de marketing: la Fórmula UEM", Revista de Docencia Universitaria. REDU. Vol. 10 (1), pgs. 199214.

CANO, M.E. (2008). "La evaluación por competencias en la educación superior", Universitat de Barcelona. Disponible en línea: http://www.ugr.es/local/recfpro7rev123COL1.pdf. Última fecha de acceso: 2 de abril 2013.

DE JUAN, M.D.; GONZÁLEZ, E.; PARRA, J.F.; KANTHER, A. Y SARABIA, F.J. (2008)."Antecedentes del aprendizaje autorregulado del estudiante universitario de marketing", Actas del XX Encuentro de Profesores Universitarios de Marketing, 17-19 de septiembre de 2008, Gran Canaria.

ESPINOSA, J.; JIMÉNEZ, J.; OLABE, M. Y BASOGAIN, Y. (2006). "Innovación docente para el desarrollo de competencias en el EEES", Universidad del País Vasco. Disponible en la web: http://campus.usual.es/ofees/ARTICULOS/p216.pdf. Última fecha de acceso: 2 de abril 2013.

FELDER, R.M. Y SILVERMAN, L.K. (1988). "Learning teaching styles in engineering education", Engineering Education, 78 (7), pp. 674-681.

GAZZANIGA, M., BOGEN, J.E. Y SPERRY, R.W. (1962). "Some functional effects of sectioning of the cerebral commissures in man", Proc. Nat. Acad. Sci., 48, pp. 1765-1769.

GARDNER, H. (1983). Inteligencias múltiples. La teoría en la práctica. Barcelona, Paidos.

GARCÍA, A.J. Y TROYANO, Y. (2010). "Aprendizaje cooperativo en personas mayores universitarias", Revista Interamericana de Educación de Adultos, Vol. 32 (1), pgs.1-15

GIL, C.; ALÍAS, A.; MONTOYA, M.D.G. (2006). "Cómo mezclar diferentes metodologías docentes para motivar e impulsar a un mayor número de alumnos", VI Jornadas de Aprendizaje Cooperativo, Barcelona.

GÓMEZ (2003). "Identificación de los estilos de aprendizaje en estudiantes de magisterio en ciencias de la educación de la Universidad de Cádiz", Revista Electrónica Interuniversitaria de Formación de Profesorado, 14, pp. 2-6.

GÓMEZ, A., RECIO, R.G., GÓMEZ, M.A. Y LÓPEZ, H. (2010). "Diagnóstico de estilos de aprendizaje en estudiantes universitarios de nuevo ingreso basado en la dominancia cerebral", Revista Estilos de Aprendizaje, 5 (5), pp. 1-17.

GONZÁLEZ, N. Y GARCÍA, Ma. R. (2007). "El aprendizaje cooperativo como estrategia de enseñanza-aprendizaje en Psicopedagogía (UC): 
repercusiones y valoraciones de estudiantes", Revista Iberoamericana de Educación, Vol. 42 (6), pgs. 1-13.

HERRMANN, S.L.M. (1989). The creative brain evolution. Búfalo, Brain Books.

JIMÉNEZ, C.A. (2006). Diagnóstico teoría del cerebro total. Perieda, Magisterio.

JOHNSON, D.W. Y JOHNSON, R.T. (1989). "Cooperative learning: What special education teachers needs to know", Pointer, Vol. 33 (2), pgs. 5-10.

KOLB, D. (1984). Experiential learning. Englewood Jffs, New York, Prentice Hall.

KOLB, D. Y KOLB, A. (2005). "Learning styles and learning spaces: Enhancing experiential learning in higher education", Academy of management Learning and Education, 4 (5), pp. 193-212.

MACLEAN, P. (1990). The triune brain evolution, New York, Planum Press.

PÉREZ, J. (2001). "Programación Neurolinguística y sus estilos de aprendizaje". Disponible en http://www.aldeaeducativa.com laldea/tareas2.asp?which=1683, marzo de 2014.

PUJOLÀS, P. (2008): 9 ideas clave. Aprendizaje cooperativo, Barcelona, Editorial Grao.

PULIDO DE BAZÁN, M.S. Y CONTRERA, G. (2005): "Análisis de los diferentes estilos de aprendizaje de los alumnos del último año de carrera de ingeniería agronómica para ajustar la metodología de enseñanza", Revista del CIZAS, Vol. 6, pgs.18-30.

SALAS, R., REGO, S. Y PARRA, A. (2004). "Enfoques de aprendizaje y dominancias cerebrales entre estudiantes universitarios", Revista Aula Abierta, 84, pp. 3-22.

SPERRY, R.W. (1973). "Cerebral organization and behavior: The Split brain behaves in many respects like two separate brains providing new research possibilities", Science, 2 (133), pp. 1749-1757.

VELÁSQUEZ, B., REMOLINA, N. Y CALLE, M.G. (2007). "Determinación del perfil de dominancia cerebral o formas de pensamiento de los estudiantes del primer semestre del programa de bacteriología y laboratorio clínico", Nova, 5 (7), pp. 48-56.

VILES, E., ZÁRRAGA-RODRÍGUEZ, M. Y JACA, C. (2013): "Herramienta para evaluar el funcionamiento de los equipos de trabajo en entornos docentes", Intangible Capital, Vol. 9 (1), pgs. 281-304. 